\title{
SimHumalator: An Open Source End-to-End Radar Simulator For Human Activity Recognition
}

\author{
Shelly Vishwakarma*, Wenda Li $^{*}$, Chong Tang*, Karl Woodbridge ${ }^{\dagger}$, Raviraj Adve ${ }^{\ddagger}$, Kevin Chetty* ${ }^{*}$ Department \\ of Security and Crime Science, University College London, $\mathrm{UK}^{\dagger}$ Department of Electronic and Electrical \\ Engineering, University College London, $\mathrm{UK}^{\ddagger}$ Department of Electrical and Computer Engineering, \\ University of Toronto, Canada \{s.vishwakarma, wenda.li,chong.tang.18, k.woodbridge, k.chetty\}@ucl.ac.uk, \\ rsadve@comm.utoronto.ca
}

\begin{abstract}
Radio-frequency based non-cooperative monitoring of humans has numerous applications ranging from law enforcement to ubiquitous sensing applications such as ambient assisted living and bio-medical applications for non-intrusively monitoring patients. Large training datasets, almost unlimited memory capacity, and ever-increasing processing speeds of computers could drive forward the data-driven deep-learningfocused research in the above applications. However, generating and labeling large volumes of high-quality, diverse radar datasets is an onerous task. Furthermore, unlike the fields of vision and image processing, the radar community has limited access to databases that contain large volumes of experimental data. Therefore, in this article, we present an open-source motion capture data-driven simulation tool, SimHumalator, that can generate large volumes of human micro-Doppler radar data in passive WiFi scenarios. The simulator integrates IEEE 802.11 WiFi standard(IEEE 802.11g, $n$, and ad) compliant transmissions with the human animation data to generate the micro-Doppler features that incorporate the diversity of human motion characteristics and the sensor parameters. The simulated signatures have been validated with experimental data gathered using an in-house-built hardware prototype. This article describes simulation methodology in detail and provides case studies on the feasibility of using simulated micro-Doppler spectrograms for data augmentation tasks.
\end{abstract}

Index Terms-Passive WiFi Sensing, micro-Doppler, activity recognition, deep learning, simulator

\section{BACKGROUND}

Recent years have witnessed significant growth in the proportion of the aging population and the number of people with long-term disability and chronic health conditions. According to World population prospects 2019, it is expected that by 2050 there will be one person in every six over the age of 65 , which is $16 \%$ higher compared to 2015 [1]. The growth in these numbers has already distressed the existing health care systems worldwide due to increased demand for dedicated residential care services that require continuous close monitoring of the patients and older people. Therefore, there is an urgent need for technologies that enable healthy living to ensure the health,

Authors addresses: S. Vishwakarma*, W Li*, C Tong*, K Chetty* are with Department of Security and Crime Science, University College London, UK E-mails: \{s.vishwakarma, wenda.li,chong.tang.18, k.chetty\}@ucl.ac.uk. (Corresponding author: Shelly Vishwakarma.)

$\mathrm{K}$. Woodbridge ${ }^{\dagger}$ is with Department of Electronic and Electrical Engineering, University College London, UK. E-mail: k.woodbridge@ucl.ac.uk.

Raviraj Adve ${ }^{\ddagger}$ is with Department of Electrical and Computer Engineering, University of Toronto, Canada. E-mail: rsadve@comm.utoronto.ca. safety, and well-being of people of all age groups, with a particular focus on supporting people living independently in their own homes.

There is considerable interest in developing cost-effective and reliable technologies enabling healthy living. The technologies are specifically tailored for the elderly population, catering to their special needs of non-invasive continuous monitoring while offering them complete freedom to live a fulfilling life. The increased research interest in these innovative technologies is evoked by their likely contribution to the efficiency and effectiveness of future health care. The traditional sensing technologies use intrusive wearable sensors such as wrist-worn units acting as emergency rescue buttons to handle emergencies at home or outside [2]. However, the usage of wearable sensors and expensive camera systems is limited because they can operate only under suitable environmental conditions and are affected by perceived privacy risk, comfort level, and battery life. Moreover, the cameras can work only in the line of sight conditions and thus have limited coverage [3]. X-ray signals possess good wall penetration capability. However, the technology is bulky and expensive, and the radiation can potentially harm the patient. Fortunately, radio frequency (RF) sensing being non-invasive can elevate the need for in-person monitoring services. It can work even in nonline-of-sight scenarios (mainly through-the-wall scenarios), making it a safe alternative. RF sensing is low-cost, noninvasive, privacy-preserving, and can continuously monitor human activities remotely using commercial off-the-shelf components (COTS) [4], [5].

Humans are rarely still. When illuminated by a radiofrequency (RF) source, the human motions introduce a Doppler shift to the radar signals. In addition, Micromotions due to the motion of hands and legs give rise to additional frequency variations known as micro-Doppler. The micro-Doppler possesses unique and discriminative features when observed in joint time-frequency space [6]. Extensive research has exploited these signatures for indoor healthcare applications such as assisted living (for fall detection in older adults) [5], [7], bio-medical applications (for non-intrusively monitoring patients), and detecting the debilitating motor symptoms of Parkinson's patients [8].

Micro-Doppler data have been generally gathered through actual measurements using active and passive 
RF sensors [6], [9], [10]. Active sensing uses the system's transmissions as the source of illumination, whereas passive sensing leverage existing opportunistic signals such as $\mathrm{WiFi}$ (or other wireless) communications to capture RF returns passively. Due to the number of advantages, such as its receive-only nature, low energy consumption, and presence of multiple illuminators, the passive sensing systems have become increasingly popular and are extensively used for short-range indoor monitoring applications [11]-[13]. The essential functionality in all these works has been classifying human activities based on their micro-Doppler signatures. More recently, there has been spurred growth in the usage of deep learning-assisted solutions in radar signal processing due to the greater availability of memory capacity and ever-increasing processing speeds of computers [?], [14][16]. The performances of these algorithms are generally tied to a large amount of high-quality training data. However, the volumes of data captured are often limited and unbalanced due to the following reasons: First, collecting real-world micro-Doppler data can be laborious and costly. Second, various environmental conditions, sensor parameters, and target characteristics affect the performance of the data, affecting deep learning algorithms' performance. Therefore, it becomes necessary to simulate radar returns in indoor sensing scenarios that would generate large volumes of training data. The simulation data can be used to preliminary evaluate different algorithms and study the effects of radar phenomenology. It can provide a form of virtual prototyping and move in stages towards a real system. The benefits of this incremental 'divide and conquer 'approach include the fact that the performance can be investigated safely for a range of operating conditions.

There exist multiple methods to simulate human microDoppler data. The earliest method models the human leg as a double pendulum structure [17]. However, this model does not simulate radar returns from other human body parts such as the torso and arms, contributing significantly to the micro-Doppler returns. The second method uses a human walking model derived from biomechanical experiments [18]. Here, twelve analytical expressions govern the motion trajectories of 17 reference points on the human body as a function of the human's height and relative velocity. Finally, the work in [19] presents a similar analytical model-driven simulation tool to characterize the radar cross-section of a pedestrian in a near field. However, this approach is based on a constant velocity model. Therefore, it cannot capture variations in more complex motions such as falling, sitting, jumping.

The third technique uses animation data from motion capture systems to model more realistic and complex human motions. There are two types of motion capture technology available- marker-based and marker-less. The real advantage of using marker-based technology is capturing more accurate, more realistic, and complex human motions. Several markers are placed on the live actor's body parts, such as the head, torso, arms, and legs, to capture their three-dimensional time-varying positions in space in a marker-based motion capture system. Although the marker- based system is accurate, the hardware restriction limits it to a single laboratory environment. Additionally, wearing a bodysuit fitted with LED markers further increases the overhead in the data collection process, limiting the massive collection of data and various investigation scenarios. The authors of [20] first presented a complete end-to-end active radar simulator of humans using a marker-based motion capture technique. The radar scatterings were simulated by integrating the animation data of humans with primitive shapes-based electromagnetic modeling. Alternatively, the authors of [21], [22] gathered animation data using a marker-less motion capture technology based on Microsoft's Kinect. The Kinect sensors are relatively compact, easy to carry, and set up to capture data compared to the marker-based system. Freely available databases of motion capture data from CMU, University of Pennsylvania, and Ohio State are available at [23]-[25].

While previous works present an active radar simulator for generating target returns, no simulation tool exists for generating radar returns in passive WiFi scenarios. Moreover, none of the simulators presented in the prior works are publically available. Therefore, there is no means of generating synthetic databases that can augment otherwise limited measurement data and address the cold start problem in radars. In response to open science practices accelerating, improving, and accumulating scientific knowledge for others to re-use and build upon, we have publically released the animation data-driven simulation tool capable of modelling the human radar signatures in passive WiFi radar (PWR) scenarios. It will assist the sensing communities in generating large volumes of high-quality and diverse radar datasets and benchmark future algorithms. The simulator's development will reduce the labour and expense of field testing by imitating a real-world system under different operating conditions such as environmental conditions, sensor parameters, and human characteristics. More importantly, the human micro-Doppler data generated using the simulator can be used to augment limited experimental data. The simulator's reliability is improved by validating it with experimental data gathered using an in-house-built hardware prototype. The standalone executable file for the simulator is available on https://uwsl.co.uk/.

To summarize, our contributions in this paper are the following:

1) We release SimHumalator, as a standalone app publically available on https://uwsl.co.uk/. SimHumalator uses a highly modular radar simulation framework. It allows users to select different target parameters, radar parameters, and signal processing parameters. The distribution package of the SimHumalator consists of executable files and documentation files comprising a quick-start guide and reference guide.

2) While previous works present an active radar simulator to generate target returns, SimHumalator generates target returns in PWR scenarios. The simulator uses animation data from a human subject for various activities and combines it with IEEE 802.11 standard WiFi transmissions to simulate reflected signals. Pas- 
sive WiFi sensing uses cross-correlation-based processing to determine range and Doppler information from target reflections.

3) SimHumalator can generate micro-Doppler spectrograms of a human corresponding to three standard waveforms-IEEE $802.11 \mathrm{~g}$ standard at $2.4 \mathrm{GHz}$, IEEE 802.11ax standard at 5.8GHz, and IEEE 802.11ad standard at $60 \mathrm{GHz}$. The sub-6GHz WiFi signals (IEEE 802.11a, b, and n) can be used to track and monitor indoor occupants for either surveillance or healthcare purposes. On the other hand, IEEE 802.11ad wireless transmissions at $60 \mathrm{GHz}$ can be used to sense finegrained motions.

4) The signatures generated by SimHumalator is experimentally validated using an in-house-built hardware prototype.

5) Our latest development on the simulator allows users to generate a massive signature library of human micro-Doppler signatures across different target motion characteristics (aspect of the target to the radar, initial position in space, different type of motions), sensor parameters (different PWR radar configurations-monostatic, bistatic-in line, and bistatic circular, waveforms) and radar signal processing parameters (such as coherent processing interval (CPI), and packet repetition interval (PRI)) with a single click.

6) The case studies presented in this work demonstrate the feasibility of using SimHumalator to generate vast amounts of a human micro-Doppler database comprising high-quality micro-Doppler spectrograms for activity recognition applications.

7) Additionally, the study demonstrates that the synthesized signatures can be used for data augmentation purposes to solve the practical problem associated with insufficient or unbalanced micro-Doppler training data.

\section{SimHumalator}

This section presents a user-friendly, publicly available end-to-end simulation tool, SimHumalator, for generating human micro-Doppler radar signatures in WiFi-based passive radar scenarios. The radar scatterings are simulated by integrating the animation data of humans with IEEE 802.11 complaint WiFi transmissions to capture features that incorporate the diversity of human motion characteristics and the sensor parameters.

SimHumalator has a highly modular radar simulation framework. It allows users the freedom to select different target parameters, radar parameters, and signal processing parameters, as shown in Fig.1. We MATLAB's app designer to create SimHumalator's standalone application shorturl. at/acxMZ. MATLAB allows users to create a standalone desktop application and share it royalty-free with other users that do not have MATLAB installed on their systems. The SimHumalator's package includes- an executable file that installs the packaged application, files including the animation data files that enable the use of the application, and the documentation files comprising a user's quick-start guide and reference guide. The app can be installed on machines with or without MATLAB installed. It downloads the MATLAB run-time compiler from the Internet if it is not included in the installer at the packaging time (for users who do not have MATLAB installed on their machines). We restrained ourselves from including the run-time installer in the same package as the total file size became too large. Instead, we choose to generate an installer that downloads the MATLAB run-time from the web and installs it along with the deployed MATLAB application. We urge users always to install the MATLAB run-time compiler when prompted in the app installation process unless they have the same version of the MATLAB installed on their machines. The distribution package of the SimHumalator release can be downloaded from https://uwsl.co.uk/. The current release of the simulator can synthesize human radar returns for two types of radar sensors- frequency modulated continuous wave (FMCW) radar and passive WiFi radar (PWR). However, the discussion here is limited to PWR SimHumalator.

The simulator's capability to generate a diverse set of human micro-Doppler signatures for different radar, target, and radar signal processing parameters is briefly presented in the following sections.

\section{A. Radar Simulation Framework}

A typical passive WiFi sensing setup is shown in Fig. 2. It comprises a reference and surveillance antenna and a signal processing unit. The reference antenna is a directional antenna that captures the direct signal from the WiFi access point (AP). On the other hand, the surveillance antenna is omnidirectional to capture the reflected signals of the human targets present anywhere in the sensing area. The signals reflected off the targets are time-delayed and Doppler-shifted direct signals. The radar signal processing unit aims to estimate these parameters using direct and reflected signals.

SimHumalator integrates the IEEE 802.11 standard compliant WiFi signal and the human animation data to generate the radar scatterings off the humans. The hybridization methodology is depicted in Fig. 3. The target reflections are simply the time-delayed and Doppler-shifted replica of the direct WiFi transmissions. The time delay is directly proportional to the target range, Doppler shift to the target's velocity, and the complex reflectivity to the target's size, shape, and material. The direct and the reflected signals are then cross-correlated in the delay-Doppler plane to generate the cross ambiguity function (CAF).

\section{1) WiFi Signal Model}

SimHumalator uses MATLAB's WLAN toolbox to simulate three IEEE standard-compliant waveforms- IEEE 802.11g at 2.4GHz, IEEE $802.11 \mathrm{ax}$ at $5.8 \mathrm{GHz}$, and IEEE $802.11 \mathrm{ad}$ at $60 \mathrm{GHz}$. The physical layer of IEEE 802.11 standards uses a packet-based protocol where each transmission packet (a physical layer conformance procedure (PLCP) protocol data unit (PPDU)) comprises a preamble and data as 


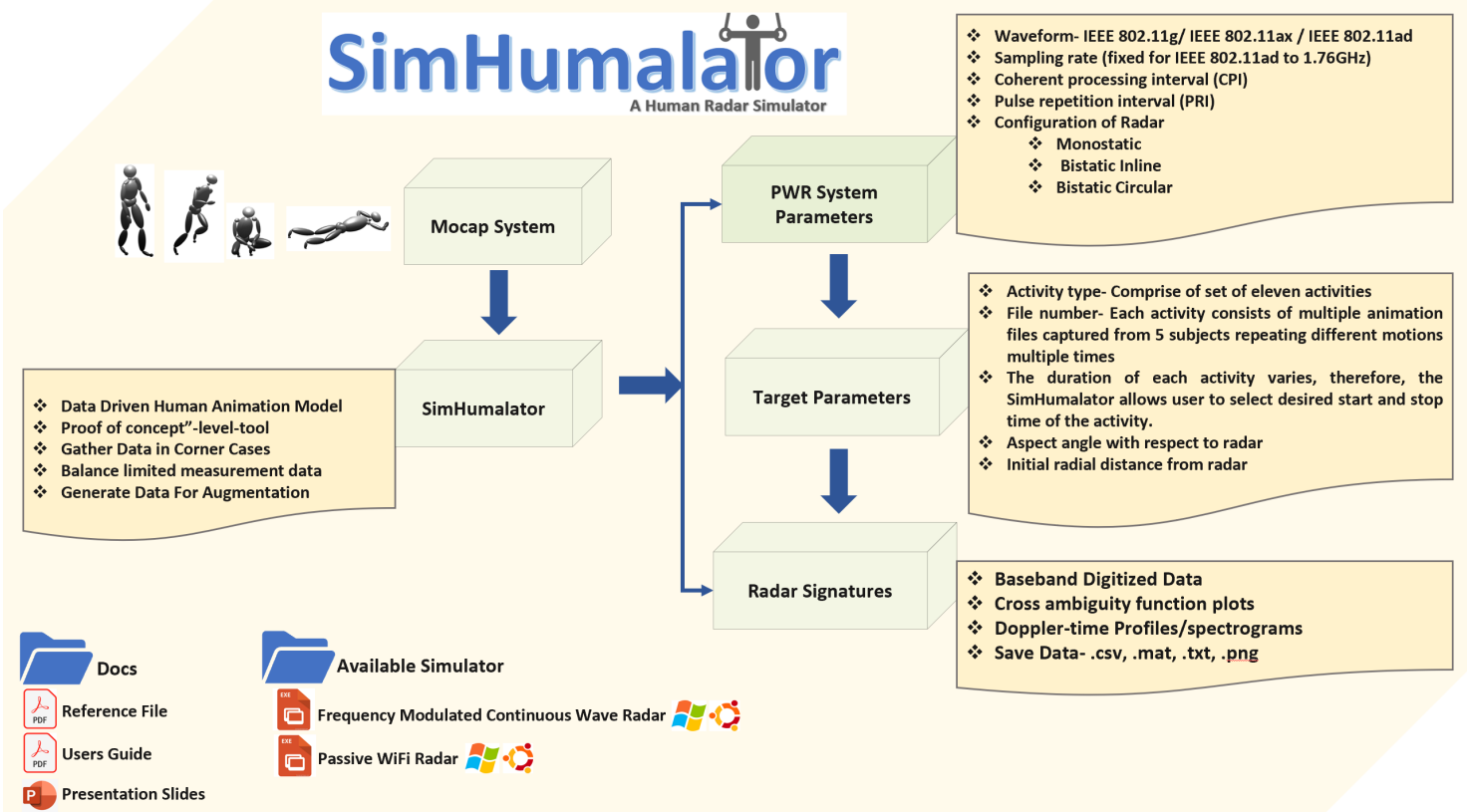

Fig. 1: SimHumalator's distribution package.

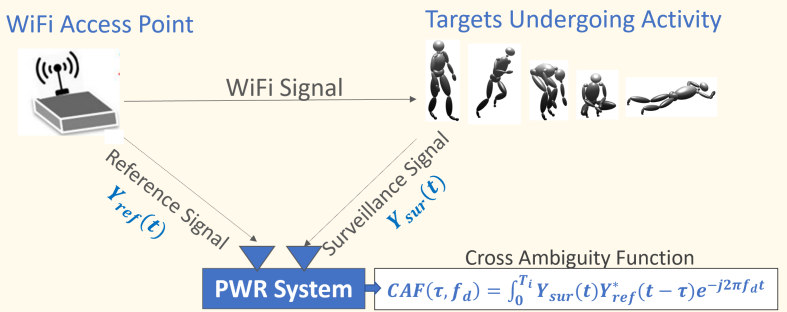

Fig. 2: A typical passive WiFi radar scenario comprising of transmissions from WiFi access points and targets undergoing motion in the same propagation environment

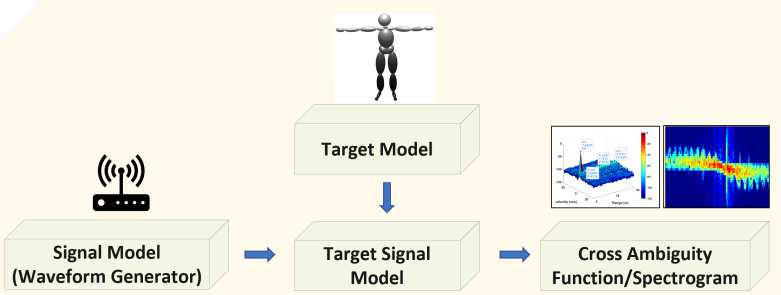

Fig. 3: Radar signal model after integration of the target model with WiFi transmissions

shown in Fig. 4. The preamble field is embedded with three sub-fields, each comprising of several time-domain samples in legacy short training field (L-STF), legacy long training field (L-LTF), and legacy signal field (L-SIG). L-STF possesses excellent correlation properties and is therefore used to detect the start of the packet, L-LTF field for communication channel estimation, and the third preamble field L-SIG to indicate the amount of data transmitted (in octets). On the other hand, the data field contains user

\begin{tabular}{|c|c|c|c|}
\hline L-STF & L-LTF & L-SIG & DATA \\
\hline 180 & $180^{-1}$ & $60^{--}$ & \multirow[t]{2}{*}{$27600=879$ Octets } \\
\hline & Preamble & & \\
\hline
\end{tabular}

Fig. 4: IEEE 802.11g standard complaint OFDM transmission packet structure

payload, medium access control (MAC) headers, and cyclic redundancy check (CRC) bits. We use only the preamble bits to form our discrete-time sequence $y_{R e f}[n]$. The simulated IEEE 802.11g packet structures mimic real WiFi transmission formats at the $2.4 \mathrm{GHz}$ band with a channel bandwidth of $B W=20 \mathrm{MHz}$. In comparison, IEEE 802.11ax and IEEE 802.11ad packet structures mimic real WiFi transmission formats at $5.8 \mathrm{GHz}$ and $60 \mathrm{GHz}$. The channel bandwidth for IEEE 802.11ad WiFi transmissions is fixed to $1.76 \mathrm{GHz}$, while the bandwidth for IEEE 802.11ax transmissions can be chosen between $B W=20 \mathrm{MHz}, 40 \mathrm{MHZ}, 80 \mathrm{MHz}, 160 \mathrm{MHz}$.

A general formulation of WiFi transmission signal $y_{\text {Ref }}(t)$ is shown in (1). It comprises of a continuous stream of $P$ transmission packets each of duration $T_{P}$ sec at a carrier frequency of $f_{C}$ as shown in Fig. 5.

$$
Y_{R e f}(t)=\frac{1}{\sqrt{(}(P)} \sum_{p=1}^{P} \sum_{n=1}^{N} y_{R e f}[n] e^{-j 2 \pi \delta f\left(t-p T_{P}\right)} e^{-j 2 \pi f_{c} t}
$$

$T_{P}$ corresponds to the packet repetition interval which is equal to pulse repetition interval in our case, $N$ is the total number of time-domain samples in one transmission packet, $t_{s}=(1 / B W)$ sec is the sampling period and $\delta f$ is OFDM sub-carrier spacing. We have used uniform packet repetition interval for our simulations. However, the future development of the simulator would include transmissions 


PuRI: Pulse Repetition Interval
PRI: Packet Repetition Interval
D: Used Data Bits

Fig. 5: IEEE 802.11g standard complaint continuous packet transmission.

of packets with staggered packet repetition interval that is the transmission of packets with different data payloads.

\section{2) Dynamic Target Model}

SimHumalator uses the animation files gathered using two motion capture technologies- a marker-based motion capture system called PhaseSpace and a marker-less Microsoft's Kinect v2 sensor system. The real advantage of using motion capture technology is capturing more accurate, more realistic, and complex human motions. The data include three-dimensional time-varying skeletal information of the human body, including 25 joints such as head center location, knee joints, elbow joints, and shoulder joints. It assumes the radar scattering centers are lying on the center of the bones, resulting in 19 scatterers on the human body. The adopted simulation methodology is presented in Fig. 6 .

The human skeleton is embodied with elementary shapes to model different body parts, such as the torso, arms, and legs using ellipsoids and the head using a sphere. The radar scattering centers are assumed to be lying approximately at the center of these primitive shapes. SimHumalator uses this data to gather time-varying range $r_{b}(t)$ and Doppler information of $f_{D b}(t)$ of each scatterer. It then computes the reflectivities $a_{b}(t)$ of each of these $B$ primitive shapes and take into account various factors such as the aspect angle $\theta_{b}(t)$, and the relative position $r_{b}(t)$, of the scattering center on the primitive shape with respect to the radar.

The reflectivity of a primitive $b$, at any time instant $t$ is given by

$$
a_{b}(t)=\frac{\zeta(t) \sqrt{\sigma_{b}(t)}}{r_{b}^{2}(t)}
$$

Here, $\zeta(t)$ subsumes propagation effects such as attenuation, antenna directivity, processing gains, $\sigma_{b}(t)$ is the radar cross section of the primitives. The RCS of primitive shapes are well characterised at microwave frequencies [26]. The RCS of an ellipsoid of length $L_{b}$ and radius $R_{b}$ is given by

$$
\sigma_{b}(t)=\sqrt{\Gamma} \frac{\frac{\pi}{4} R_{b}^{4} L_{b}^{2}}{R_{b}^{2} \sin ^{2} \theta_{b}(t)+\frac{1}{4} L_{b}^{2} \cos ^{2} \theta_{b}(t)}
$$

The dielectric properties of human skin are incorporated into the RCS estimation through the Fresnel reflection coefficient $\Gamma$. The human is assumed to be a single-layer dielectric with a dielectric constant of 80 and conductivity of $2 \mathrm{~S} / \mathrm{m}$.

\section{3) Hybrid electromagnetic radar scattering from dynamic humans}

The received signal is simply the attenuated, timedelayed $\tau_{b}$, and Doppler-shifted $f_{D b}$, versions of the direct transmitted signal $Y_{\text {Ref }}(t)$. Ignoring multipath, the baseband received signal on the surveillance channel can be represented as

$$
Y_{S u r}(t)=\sum_{p=1}^{P} \sum_{b=1}^{B} a_{b}(t) Y_{R e f}\left(t-\tau_{b}-p T_{p}\right) e^{-j 2 \pi f_{D b} p T_{p}}+z(t)
$$

Here, $c=3 \times 10^{8} \mathrm{~m} / \mathrm{s}$ is the speed of light and $z(t)$ is the additive circular-symmetric white noise.

\section{4) CAF processing}

The cross ambiguity function processing is implemented over the received data and the direct reference signal data to compute the delay $\tau_{b}$ and Doppler information $f_{D b}$ of the target. The adopted CAF processing is shown in Fig. 7. Match-filtering is performed along the fast time samples and fast Fourier transform (FFT) along the slow time samples to generate CAFs. The CAF processing is implemented as

$$
C A F\left(\tau, f_{d}\right)=\int_{0}^{T_{i}} Y_{s u r}(t) Y_{r e f}^{*}(t-\tau) e^{j 2 \pi f_{d} t} d t
$$

Where $*$ denotes complex conjugation, $\tau$ is the time delay, $f_{d}$ is the Doppler shift and $T_{i}$ is the integration time.

Note that the direct signal component is always strong and can mask the target returns present in the CAFs. Therefore, the CLEAN algorithm is used to remove direct signal interference [9]. The CLEAN algorithm subtracts the self ambiguity function (generated using only the reference signal) from the CAF calculated in (5) thereby suppressing stronger direct signals.

Multiple CAFs spanning a duration of $T_{\text {Total }}$, are processed to generate the Doppler-time spectrogram as shown in Fig. 8. Here, for each CPI, the peaks along the range axis are coherently added for each Doppler bin. 


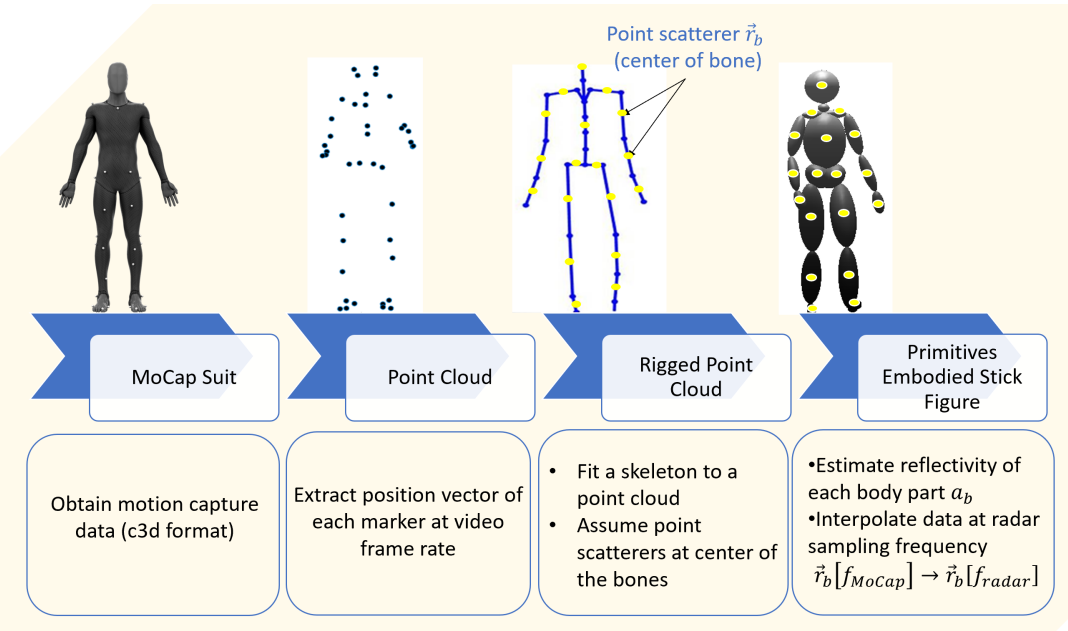

Fig. 6: Simulation framework for generating an electromagnetic human primitive scattering model from motion capture animation data.
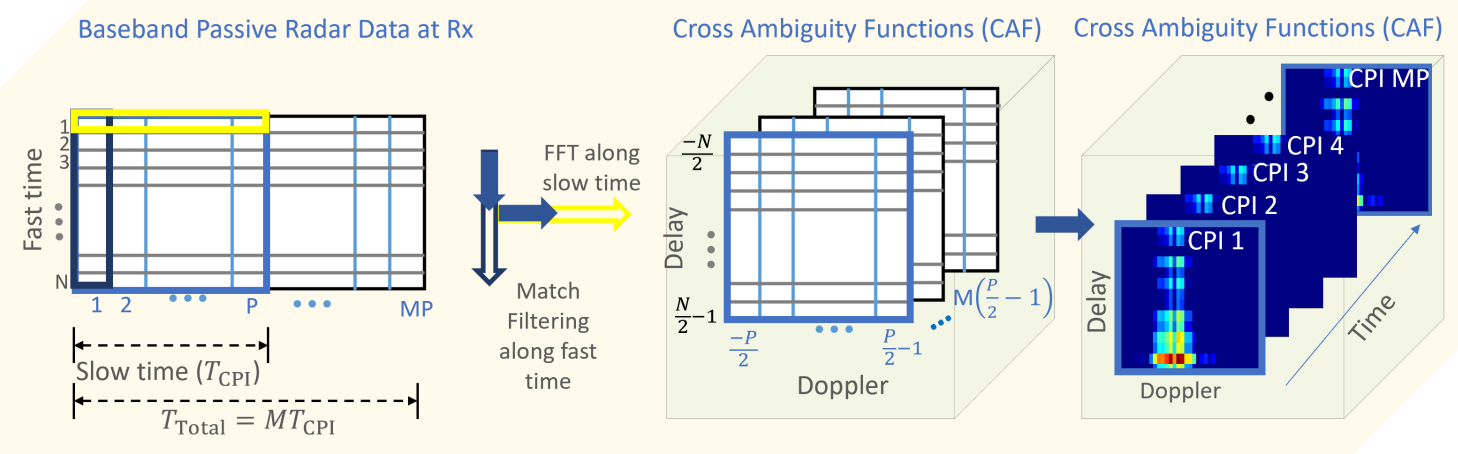

Fig. 7: cross ambiguity function generation through cross-correlation between the direct WiFi transmission and the reflected signals off the targets

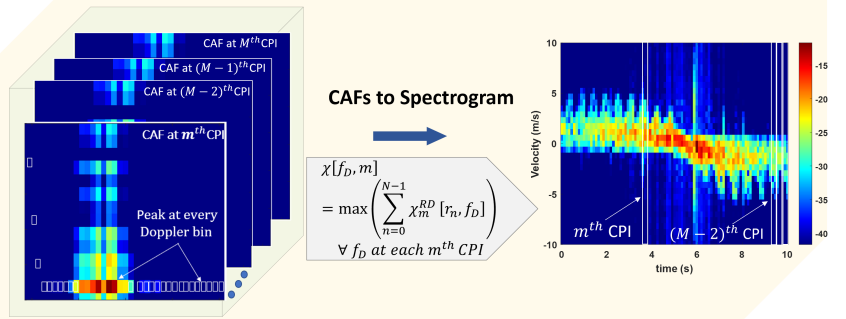

Fig. 8: Simulation methodology to generate micro-Doppler spectrograms using multiple CAFs spanning the entire duration of motion

\section{B. Graphical User Interface}

SimHumalator allows the user to select many input parameters to specify the target motion characteristics, sensor operating conditions, and signal processing parameters. The simulator receives the simulation inputs with the help of a GUI window, as shown in Fig.9. In addition, the user's manual can help visualize the parameters within the simulation inputs window represent and how changing these parameters affects the radar signatures.

SimHumalator can generate a massive signature library of radar signature profiles of various human activities, including cross-ambiguity functions and Doppler-time plots. Since we are simulating IEEE 802.11g WiFi transmissions, the signal bandwidth is limited to $20 \mathrm{MHz}$, insufficient to locate targets in most indoor scenarios. Therefore, we focus on extracting only the time-varying micro-Doppler information in joint time-frequency space. Moreover, the standard waveforms IEEE 802.11ax and IEEE 802.11ad, which offer a good resolution range, do not comprise complementary codes that hold perfect autocorrelation properties. Therefore, cross-correlation operation results in high sidelobe levels significantly affecting the quality of range-time signatures. Even if the waveforms have perfect autocorrelation properties, the sidelobe levels are zero only for static target scenarios. As soon as the target starts moving, high sidelobes appear in the range domain.

SimHumalator offers users the freedom to select animation data files from eleven healthcare-related human motion classes. These include human rotating his body (HBR) while standing in a fixed position, human kicking (HK), human punching (HP), human grabbing an object (HG), human walking back and forth in front of the radar (HW), human standing up from a chair (HStand), human 


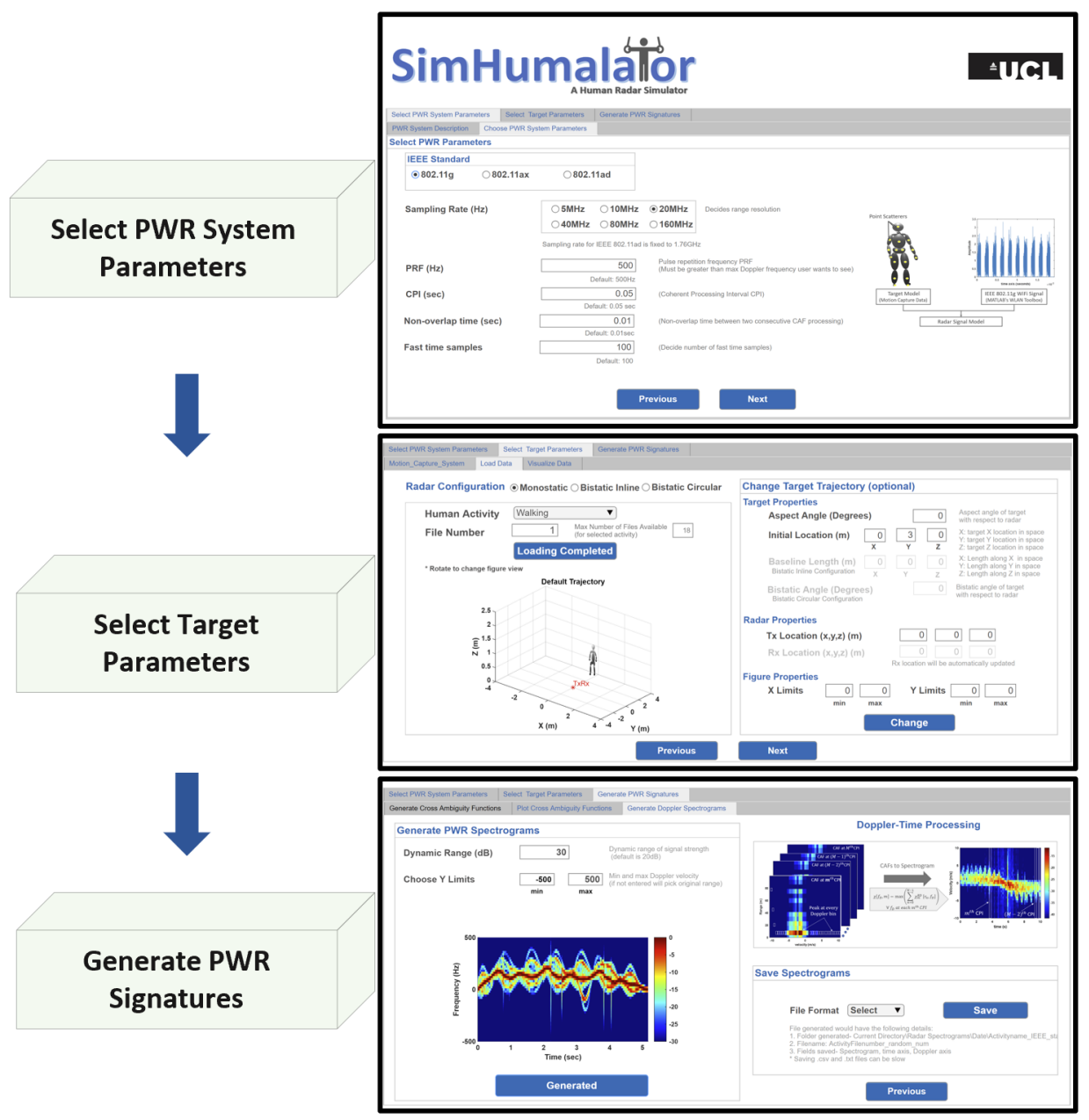

Fig. 9: SimHumalator's graphical user interface for choosing PWR system parameters

TABLE I: Motion capture data information

\begin{tabular}{|l|c|}
\hline \multicolumn{1}{|c|}{ Activity } & Number of files \\
\hline Human rotating his body & 104 \\
\hline Human kicking & 104 \\
\hline Human punching & 103 \\
\hline Human grabbing an object & 103 \\
\hline Human walking back and forth & 106 \\
\hline Human standing up from chair & 71 \\
\hline Human sitting down on chair & 71 \\
\hline Human stand up from chair to walk & 69 \\
\hline Human walk to sit on chair & 68 \\
\hline Human walk to fall on ground & 60 \\
\hline Human standing up from ground to walk & 60 \\
\hline
\end{tabular}

sitting down on a chair (HSit), human stand up from the chair to walk (HSW), human walk to sit on a chair (HWS), human walk to fall on the ground (HWF) and a human standing up from the ground to walk (HFW). The number of animation files for each of these activities is presented in Table I.

Some files of the first five activities- HBR, HK, HP, HG, and HW, have been captured before the Covid-19 pandemic using marker-based active tracking Phase-Space system [27]. The remaining six activities and some data for the first five activities were captured using Kinect v2 sensor during
TABLE II: Simulated PWR Parameters

\begin{tabular}{|c|c|}
\hline Radar Parameters & Values \\
\hline Carrier frequency $\left(f_{c}\right)$ & $60 \mathrm{GHz}$ \\
\hline Bandwidth $(B W)$ & $1.762 \mathrm{GHz}$ \\
\hline Packet Repetition Frequency $\left(P R F=1 / T_{p}\right)$ & $1000 \mathrm{~Hz}$ \\
\hline Coherent Processing Interval & $0.05 s$ \\
\hline Maximum Doppler $\left(f_{D \max }= \pm P R F / 2\right)$ & $\pm 500 \mathrm{~Hz}$ \\
\hline Doppler resolution $\left(\Delta f_{D}=1 / C P I\right)$ & $20 \mathrm{~Hz}$ \\
\hline
\end{tabular}

Covid-19 pandemic [28]. We will continue to build our database and provide the user community with a platform to generate substantial radar databases.

\section{Synthesized SPECTROgRAM EVALUATION}

The PWR signal parameters used are provided in TABLE II. The CPI is fixed to $0.05 \mathrm{~s}$, which is sufficient to capture time-varying micro-Doppler features in joint timefrequency space. Note that the PRF dictates the maximum Doppler frequency range that can be observed without aliasing $\left(f_{D \max }= \pm P R F / 2\right)$, Doppler resolution by the CPI $\left(\delta f_{D}=1 / C P I\right)$, and the choice of the non-overlap time window is critical for successfully representing the microDoppler signature of a dynamic target. The non-overlap time window here is similar to the short-time window in the case of the short-time Fourier transform. The non-overlap 


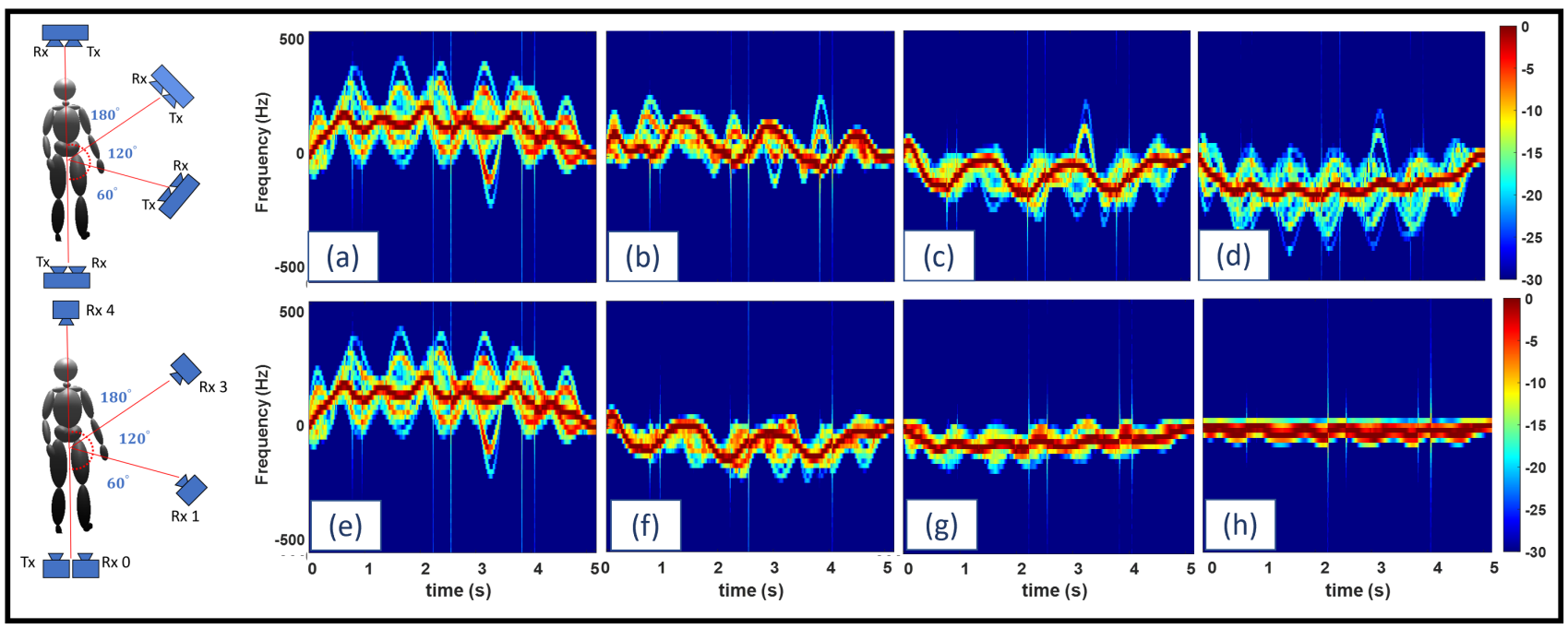

Fig. 10: (a)-(d) Radar micro-Doppler signatures of human walking at four aspect angles $0^{\circ}, 60^{\circ}, 120^{\circ}$, and $180^{\circ}$ with respect to the monostatic radar respectively. (e)-(h) Radar micro-Doppler signatures of human walking at aspect angles $0^{\circ}$ to the radar at four bistatic radar configurations with following bistatic angle $0^{\circ}, 60^{\circ}, 120^{\circ}$ and $180^{\circ}$ respectively.

time window should ideally be low for fast-moving moving targets and high for capturing motion characteristics of slow-moving targets. Therefore, users should choose these parameters carefully to obtain the desired extent and resolution spectrograms.

\section{A. Monostatic Configuration of PWR System With Varying Target Aspect Angles}

In the monostatic configuration of the PWR system, the WiFi AP and radar receiver are co-located. Fig.10(a) presents the micro-Doppler signature of a human moving at $0^{\circ}$ aspect angle with respect to the radar receiver (direct lineof-sight). Since the human is walking in the direction of radar, the micro-Doppler are mostly positive. The strongest returns arise from the torso due to its larger cross-section, while the weaker returns are generated from the motions of the arms and legs. Some of the negative Dopplers arise from the back-swing of the arms and legs. In most realistic scenarios, the human motions might not be restricted to a single aspect angle with respect to the radar. In such scenarios, the spectrograms might differ significantly. It could be due to the shadowing of some part of the human body if captured at different angles.

Fig.10(b)-(d), shows the spectrograms of a human walking at three different aspect angles- $60^{\circ}, 120^{\circ}$ and $180^{\circ}$ with respect to the radar respectively. The signatures at $60^{\circ}$ shown in Fig.10(b), mostly have reduced positive Dopplers compared to $0^{\circ}$ aspect angle. This is because the target is still approaching but with a reduced radial component towards the radar. The Dopplers become negative when the target aspect angle is $120^{\circ}$. At this angle, the target begins to move away from the radar. Finally, the signatures at $180^{\circ}$ represent a human walking away from the radar, thus having all the negative Dopplers.

\section{B. Bistatic Configuration With Varying Bistatic Angle And} Fixed Target Aspect Angle

In most passive WiFi sensing scenarios, the WiFi AP and the radar receiver are separated by a certain distance resulting in a bistatic configuration of the setup. Here, the Doppler frequency $f_{D}=\frac{2 v f_{c}}{c} \cos (\theta) \cos (\beta / 2)$, is governed by aspect angle $\theta$ and the bistatic angle $\beta$. For simplicity, the aspect angle of the target is fixed at $0^{\circ}$; therefore, the Dopplers only depend upon the bistatic angles $f_{D}=$ $\frac{2 v f_{c}}{c} \cos (\beta / 2)$. Fig.10(f)-(h) show the micro-Doppler signatures of a walking human at four bistatic angles $60^{\circ}, 120^{\circ}$ and $180^{\circ}$ respectively. In the forward scatterer position, when $\beta=180^{\circ}$, the Doppler should be zero $\left(f_{D}=0\right)$. However, since humans are extended targets, some microDoppler returns are due to the swinging motion of arms and legs. This is evident in Fig.10(h).

\section{Micro-Doppler Spectrograms Across Multiple Standards}

Growing demand of throughput for next-generation multimedia wireless services such as video streaming, data transfer amongst smart terminals (Internet of Things), highdense deployment areas such as airports, shopping malls, stadiums, and office spaces, has necessitated the need for creating complex wireless networks. Therefore, to accommodate the increasing demands and challenging application scenarios, most WiFi devices will soon be equipped with antennas operating at both sub- $7 \mathrm{GHz}$ frequencies (to be compatible with legacy standards - IEEE 802.11ax and earlier) and mmWave frequencies (60 GHz IEEE 802.11ad/ay and subsequent) [29].

The sub-7 GHz WiFi signals have better wall penetration capabilities and richer multipath information. Therefore, they can be used to sense relatively large motions even through obstacles (through walls). On the other hand, the measurements at mmWave can sense fine-grained motions and provide highly directional information through beamforming. Fig.11 presents the micro-Doppler spectrograms 


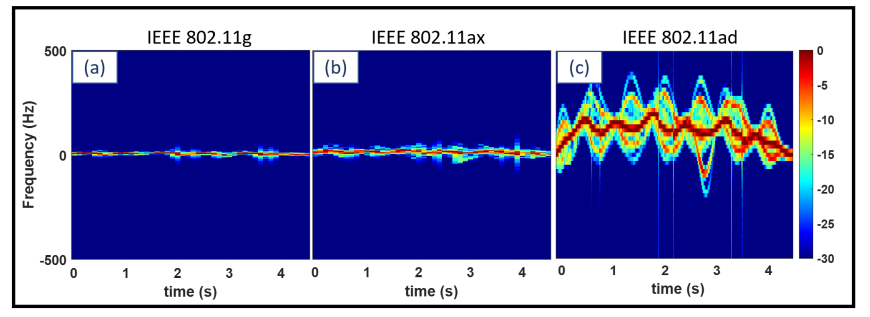

Fig. 11: Micro-Doppler spectrograms of a human walking motion for (a) IEEE $802.11 \mathrm{~g}$ standard at $2.4 \mathrm{GHz}$, (b) IEEE $802.11 \mathrm{ax}$ standard at $5.8 \mathrm{GHz}$, and (c) IEEE $802.11 \mathrm{ad}$ standard at $60 \mathrm{GHz}$ respectively.

of a human walking motion corresponding to three standard waveforms-Fig.11(a) IEEE $802.11 \mathrm{~g}$ standard at $2.4 \mathrm{GHz}$, Fig.11(b) IEEE 802.11ax standard at 5.8GHz, and Fig.11(c) IEEE 802.11ad standard at 60GHz. We keep fixed maximum detectable Doppler frequency to $\pm 500 \mathrm{~Hz}$ and non-overlap time window to $0.5 \mathrm{sec}$ for all the plots to demonstrate the substantial differences arising from different carrier frequencies. The figures demonstrate that the Doppler spectrograms corresponding to high carrier frequency data show finer frequency resolution than those from low carrier frequency data. However, the classification algorithms might pose some unique challenges when the measurement data "merge" sensing inputs from sub-7 GHz and mmWave. Therefore, to demonstrate the usefulness of multi-band WiFi sensing systems in real-world scenarios, the algorithms would need to use non-heuristic methods to derive features for handling the diversity in the measurement data.

\section{CAse Studies}

This section presents case studies to demonstrate the feasibility of using SimHumalator to generate vast amounts of a human micro-Doppler database comprising highquality micro-Doppler spectrograms for activity recognition applications. Additionally, the study demonstrates that the synthesized signatures can be used for data augmentation to solve the practical problem of insufficient or unbalanced micro-Doppler training data. Some other studies used SimHumalator for studying human micro-Doppler signature classification in the presence of a selection of jamming signals [30].

The case studies use 802.11 g packet structures to mimic real WiFi transmission formats at the $2.4 \mathrm{GHz}$ band with a channel bandwidth of $B W=20 \mathrm{MHz}$.

\section{A. Human Activity Recognition Using SimHumalator Gener- ated Spectrograms}

The human micro-Doppler spectrograms simulated from SimHumalator are classified for the following activities: human standing in a fixed position while rotating their body (HBR), human kicking (HK), human punching (HP), human grabbing an object (HG), and a human walking back and forth in front of the radar (HW). Sample spectrograms for the five motion classes are shown in Fig. 12. These motions are periodic and thus have alternating positive and negative micro-Doppler features. Therefore, it becomes challenging for any classifier to discern the correct motion class. This section studies the different classification algorithms' robustness to classify micro-Dopplers in more complex scenarios, such as varying aspect angles and bistatic angles. Note that the human motions in these repeated measurements were unrestricted, and, therefore, the micro-Doppler signatures vary due to differences in gait patterns in every simulation. The duration of each measurement was $4.5 \mathrm{sec}$. A sliding window of duration $1.5 \mathrm{sec}$ with an overlapping time of $0.5 \mathrm{sec}$ was used over the full signature of duration 4.5sec. It resulted in 9 spectrograms, each of duration $1.5 \mathrm{sec}$ from every motion capture file. TABLE III summarizes the entire simulation database.

We use handpicked features [31], Cadence velocity features (CVD) [32], and automatically extracted sparse features [33], [34] from the micro-Doppler signatures to test the performance of classical machine learning-based support vector machine (SVM) classifier [35]. We then compare their performances with a deep convolutional neural network (DCNN) that has a joint feature extraction and classification framework within the same network. We designed a 24layered deep neural network comprising three components (convolution layer, pooling layer, and activation functions). We also test some pre-trained deep neural networks such as AlexNet, GoogLeNet, and ResNet18. We used $70 \%$ of each target's spectrograms as the training data set, $15 \%$ as the validation set, and the remaining $15 \%$ as the test data set.

The following three classification scenarios were considered to give readers a better understanding of the sensitivity of the algorithm's performance to the simulation database.

- Case 1a: Train using data from a fixed zero aspect angle: The algorithms' performances using a simulation database generated for a fixed $0^{\circ}$ aspect angle of the target. Note that the five target classes considered in the study share common features in the micro-Doppler feature space because of the proximity between different motion categories. The resulting classification accuracies are presented in TABLE IV. The results demonstrate that all deep neural networks outperform the classical machine learning-based methods and achieve an average classification accuracy of $\approx 99 \%$. This is because the deep neural networks, a cascaded structure of neurons, can learn any complex function to create a decision boundary even for nonlinear data considered in the study. The classical machine learning algorithms, on the other hand, are not capable of learning these complex discerning boundaries, resulting in poor classification performances.

- Case 1b: Train using data from multiple aspect angles: Next, the performance was analysed when the algorithms were trained using a simulation database comprising micro-Doppler signatures captured at multiple aspect angles. It is a significantly more challenging and realistic scenario since no aspect angle information is available during the test phase. Note that the spectrograms used in testing have not been used during training. 


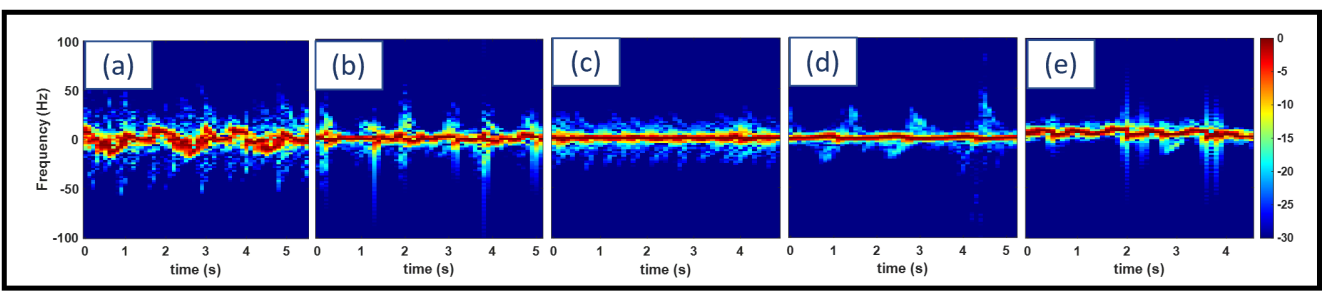

Fig. 12: Radar micro-Doppler signatures for a human undergoing (a) a body rotation motion, (b) kicking motion, (c) punching motion, (d) grabbing an object motion, and (e) walking in the direction of the monostatic configuration of PWR radar at the 2.4GHz band.

TABLE III: Simulation Dataset Description

\begin{tabular}{|c|c|c|c|c|c|}
\hline \multirow[t]{2}{*}{ Target Class } & \multirow[t]{2}{*}{$\begin{array}{l}\text { Number of MoCap } \\
\text { Files }\end{array}$} & \multirow[t]{2}{*}{$\begin{array}{l}\text { Duration of Data } \\
\text { (in each file) }\end{array}$} & \multicolumn{3}{|c|}{$\begin{array}{c}\text { Number of Spectrograms (1.5sec each) } \\
\text { In Radar Configuration }\end{array}$} \\
\hline & & & $\begin{array}{c}\text { Aspect Angle 0 } \\
\text { (Overlapping Window 0.5sec) }\end{array}$ & $\begin{array}{c}\text { Varying Aspect Angle } \\
\text { 0:5:360 } \\
\text { (No Overlapping) }\end{array}$ & $\begin{array}{c}\text { Varying Bistatic Angle } \\
\text { 0:5:360 } \\
\text { (No Overlapping) }\end{array}$ \\
\hline HBR & 10 & $4.5 \mathrm{sec}$ & 90 & 2190 & 2190 \\
\hline HK & 20 & $4.5 \mathrm{sec}$ & 180 & 4380 & 4380 \\
\hline HP & 20 & $4.5 \mathrm{sec}$ & 180 & 4380 & 4380 \\
\hline HG & 20 & $4.5 \mathrm{sec}$ & 180 & 4380 & 4380 \\
\hline HW & 19 & $4.5 \mathrm{sec}$ & 171 & 4161 & 4161 \\
\hline \multicolumn{3}{|c|}{ Total Data in Each Configuration } & 801 & 19491 & 19491 \\
\hline
\end{tabular}

TABLE IV: Classification accuracies of multiple algorithms for a simulation database (captured for a fixed aspect angle of the target)

\begin{tabular}{|c|c|c|c|c|c|c|c|}
\hline \multirow{2}{*}{ Target Class/ Algorithm } & \multirow{2}{*}{$\begin{array}{l}\text { Handpicked } \\
\text { Features }\end{array}$} & \multirow{2}{*}{$\begin{array}{c}\text { CVD } \\
\text { Features }\end{array}$} & \multirow{2}{*}{$\begin{array}{c}\text { Sparse } \\
\text { Features }\end{array}$} & \multicolumn{4}{|c|}{ Neural Networks } \\
\hline & & & & DCNN & AlexNet & GoogLeNet & ResNet18 \\
\hline HBR & 100 & 98.9 & 99.1 & 100 & 100 & 100 & 100 \\
\hline $\mathrm{HK}$ & 83.9 & 83 & 93.1 & 100 & 100 & 100 & 96.3 \\
\hline HP & 73.8 & 92.3 & 92.4 & 100 & 96.3 & 96.3 & 100 \\
\hline HG & 82.5 & 98.3 & 97.6 & 96.3 & 100 & 100 & 100 \\
\hline HW & 100 & 94.5 & 100 & 100 & 100 & 100 & 100 \\
\hline Overall Accuracy (\%) & 88 & 93.4 & 96.4 & 99.3 & 99.3 & 99.3 & 99.3 \\
\hline
\end{tabular}

TABLE V: Classification accuracies for a simulation database (captured for varying aspect angle of the target with respect to the radar receiver)

\begin{tabular}{|c|c|c|c|c|c|c|c|}
\hline \multirow{2}{*}{ Target Class/ Algorithm } & \multirow{2}{*}{$\begin{array}{c}\text { Handpicked } \\
\text { Features }\end{array}$} & \multirow{2}{*}{$\begin{array}{c}\text { CVD } \\
\text { Features }\end{array}$} & \multirow{2}{*}{$\begin{array}{c}\text { Sparse } \\
\text { Features }\end{array}$} & \multicolumn{4}{|c|}{ Neural Networks } \\
\hline & & & & DCNN & AlexNet & GoogLeNet & ResNet18 \\
\hline HBR & 93.2 & 91.6 & 98.6 & 100 & 100 & 99.4 & 100 \\
\hline HK & 41.8 & 34 & 81.7 & 94.2 & 91.8 & 88.6 & 93.6 \\
\hline $\mathrm{HP}$ & 6.1 & 90.9 & 88.1 & 95.7 & 98.3 & 93.6 & 97.6 \\
\hline HG & 29.7 & 61.5 & 90.2 & 92.5 & 89.3 & 98.5 & 99.4 \\
\hline $\mathrm{HW}$ & 90.3 & 69 & 96.5 & 87.8 & 98.6 & 99.4 & 99.8 \\
\hline Overall Accuracy (\%) & 52.02 & 69.4 & 91.02 & 94.04 & 95.6 & 95.9 & 97.84 \\
\hline
\end{tabular}

TABLE $V$ presents the resulting classification accuracies across different algorithms. The average classification accuracies are lower in this case. The reason for poor performance is that the Doppler spectrogram for a particular motion class at certain aspect angles might look similar to that for other motion classes at the same angle. Algorithms based on deep networks perform exceptionally well even under diverse training and test datasets. It indicates that these algorithms are specifically suited for problems dealing with a great deal of diversity in radar data. The best performing network is ResNet18, with an average classification accuracy of $97.8 \%$.

- Case 1c: Train using data from multiple bistatic angles: It is a more practical scenario that one can encounter. Here, the performance is observed to be lower compared to the previous two cases. This is because the micro-Doppler signatures are captured in a forward scatter geometry, resulting in micro-Doppler being centered mostly around 0 Doppler for almost all the motion classes. This leads to a significant reduction in classification accuracies. The classification results for this case are presented in TABLE VI.

- Case 1d: Performance Under Noisy Conditions Most realistic scenarios have environmental factors that significantly affect the resulting SNRs. Therefore, to assess SNR's impact on the classification performances, Gaussian noise was added to achieve varying levels of SNR (-2 to 10dB) in the simulated micro-Doppler signatures. The classification was challenging under noisy conditions due to the close similarity of motion classes considered in the dataset. Fig. 13 shows the variation of average classification performances of different algorithms as a function of SNR. As the noise level is increased, there is an expected drop in different algorithms' overall performance. However, the drop in performance is higher for classical learning-based methods (represented in black) than deep learning 
TABLE VI: Classification accuracies for a simulation database (captured under varying bistatic circular configurations)

\begin{tabular}{|c|c|c|c|c|c|c|c|}
\hline Target Class/ Algorithm & Handpicked & CVD & Sparse & \multicolumn{4}{c|}{ Neural Networks } \\
& Features & Features & Features & DCNN & AlexNet & GoogLeNet & ResNet18 \\
\hline HBR & 93.6 & 91.8 & 99.1 & 80.2 & 82.6 & 87.2 & 91.2 \\
\hline HK & 17.2 & 35.5 & 81.8 & 86.9 & 83.9 & 85.8 & 91.6 \\
\hline HP & 21.2 & 88.7 & 89.7 & 97 & 95.9 & 91.3 & 99.5 \\
\hline HG & 64.4 & 60.3 & 89.3 & 94.8 & 97.9 & 93.6 & 95.1 \\
\hline HW & 90.9 & 73.8 & 95.8 & 98.6 & 99.2 & 100 & 99.4 \\
\hline Overall Accuracy (\%) & $\mathbf{5 7 . 4 6}$ & $\mathbf{7 0 . 2}$ & $\mathbf{9 1 . 1 4}$ & $\mathbf{9 1 . 5}$ & $\mathbf{9 1 . 9}$ & $\mathbf{9 1 . 5 8}$ & $\mathbf{9 5 . 7 9}$ \\
\hline
\end{tabular}

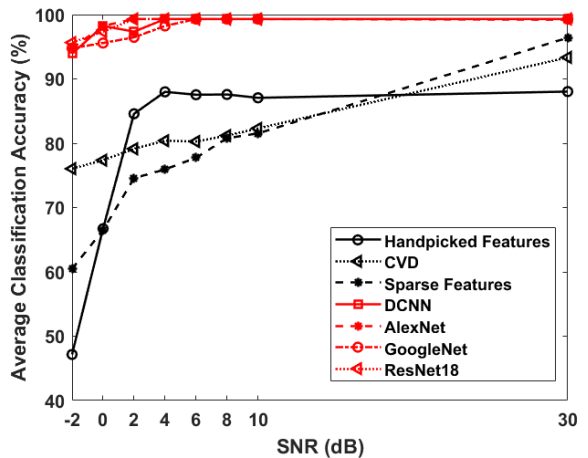

Fig. 13: Average classification accuracies as a function of SNR

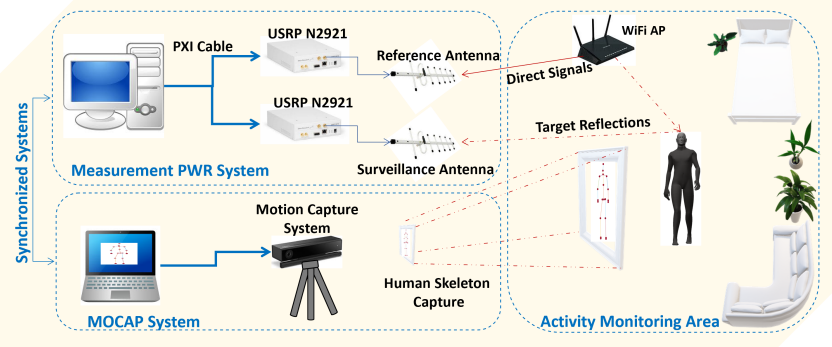

Fig. 14: The experimental setup comprising two synchronized systems- a motion capture Kinect v2 sensor and a PWR system for monitoring human activities in indoor scenarios.

models (represented in red). The results suggest that the deep networks can extract features that are more robust to noise and, therefore, be the 'classifier of choice' in high-noise scenarios (comprising various environmental factors such as noise and multipath).

\section{B. Experimental Validation of SimHumalator}

The simulator's reliability is improved by validating it with experimental data gathered using an in-house-built hardware prototype [36]. Fig. 14 presents the experimental setup comprising two synchronized systems: an infrared motion capture Kinect v2 sensor and a non-contact physical activity monitoring PWR system. The PWR system was set up using two Yagi antennas, each with a gain of $14 \mathrm{dBm}$, two National Instruments (NI) USRP-2921 [37] and a Netgear R6300 transmitter acting as the WiFi AP. The WiFi AP was configured to transmit an 802.11g standard-compliant waveform at a center frequency of $2.472 \mathrm{GHz}$. The PWR system used one antenna, a reference antenna, to capture direct WiFi transmissions from the AP. Simultaneously, the second is used as a surveillance antenna to capture signals reflected off-targets in the propagation environment. The reference WiFi signal and the surveillance signal were then cross-correlated to generate the radar micro-Doppler signatures in real-time.

Fig. 15 shows the qualitative comparison between the micro-Doppler spectrograms generated using the PWR measurement system and the SimHumalator. Fig.15 (a)-(c) shows the velocity-time profile (generated using motion capture data) and simulated and the measured spectrograms respectively for a human undergoing a motion of first sitting down on a chair followed by standing up from the chair. The qualitative similarity between all the signatures is evident from the figure. As the human sits down, there is a negative Doppler due to the bulk body motion. The positive micro-Doppler arises due to arm motion and legs moving slightly in the radar direction while sitting down. However, the strength of the micro-Doppler is low compared to bulk body motion. After a 5sec delay, the human subject stands up from the chair, resulting in primarily positive Dopplers. Similarly, Fig.15 (d)-(f) presents spectrograms corresponding to a human standing up from a chair and before walking. Fig.15 (g)-(i), presents spectrograms corresponding to a human transitioning from walking to falling. The strength of the signals of the spectrograms shown in Fig.15 (a)-(i) may not be the same; however, the envelope of the velocity-time profile is visually very similar across all. The simulations do not synthesize environmental factors like noise, propagation loss, occlusions, and multipath. Therefore, the simulated spectrograms are clean compared to the measured signatures.

\section{Experimental Data Augmentation}

SimHumalator can effectively generate high-quality micro-Doppler spectrograms from the motion capture data. Therefore, it can be used to synthesize signatures for data augmentation purposes to solve the practical problem associated with insufficient or unbalanced micro-Doppler training data [36], [38]. This section presents classification results from various data augmentation schemes. The augmentation scheme adds additional simulation data, increasing the training dataset's overall size, which only used measurement data. The study can be summarized in Fig. 16. The total measurement data $(M)$ is divided into the training and test sets according to the split ratio of $m$. Then the $s$ percentage of the total simulation data $(S)$ is added to the training set, resulting in a training set size of $(m M+s S)$.

First, the classification performance is tested when only measurement data is used for training and test scenarios. 


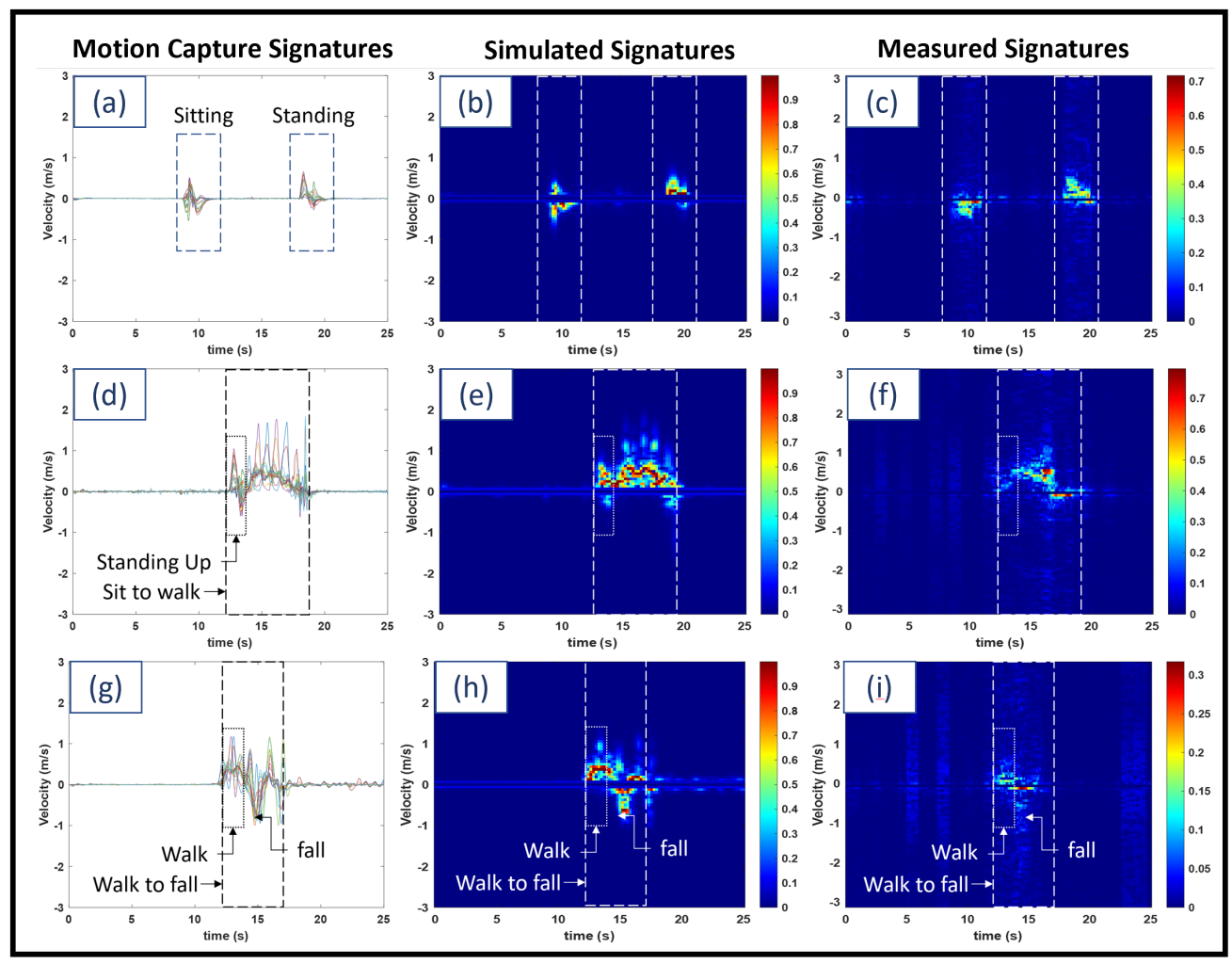

Fig. 15: Velocity-time profile using motion capture data, simulated and measured Doppler spectrograms of a human undergoing (a)-(c) sitting followed by standing up motion, (d)-(f) stand up from chair and starts walking, (g)-(i) walk to fall motion respectively.

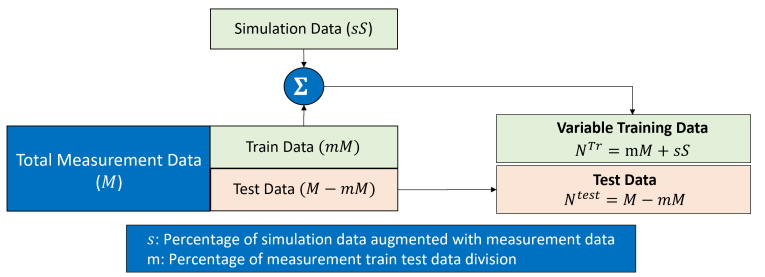

Fig. 16: Augmentation study: The training dataset changes with the percentage of simulated data (s) augmented with the measurement training data. The percentage of simulation data is varied to study the impact of data augmentation on classification performance. $s=0$ is a special case where training and test data only comprise measured spectrograms.

Next, the training dataset was created by combining the simulation and experimentally measured data. Only unseen measurement data was used to test the pre-trained VGG16 model's performance during the training process. Fig. 17 presents the augmentation results. The smaller the volume of measured data in the training set, the more pronounced the classification accuracy. The outcomes highlight that for cases where only limited experimental data is available for training, classification performance can be improved up to $8 \%$. Additionally, the data generated by the simulator can be used for replacing a small amount of low-quality measured data and filling a small number of imbalanced classes in practical applications.

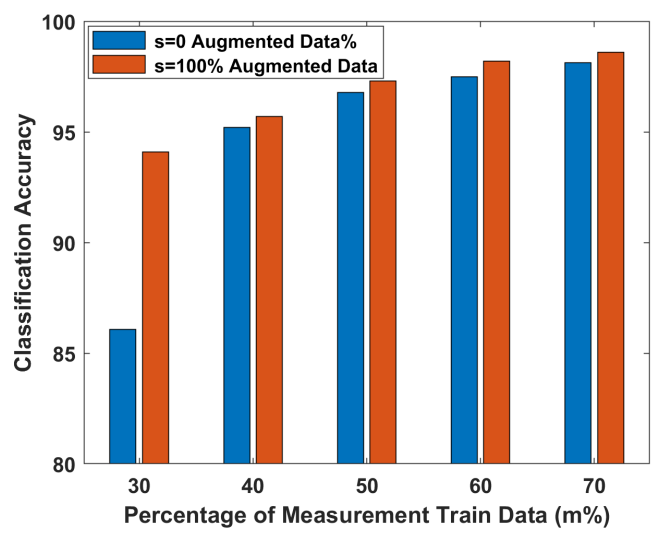

Fig. 17: Augmentation study: Classification accuracies of the percentage of measurement data augmented with simulation data $(\mathrm{s}=100 \%)$.

\section{CONCLUSION}

This work presents an open-source motion capture datadriven simulation tool, SimHumalator, that can generate large volumes of human micro-Doppler radar data at multiple IEEE WiFi standards(IEEE 802.11g, ax, and ad). SimHumalator can simulate human micro-Doppler radar returns as a function of a diverse set of target parameters, radar parameters, and radar signal processing parameters. The simulator's reliability is improved by validating it with experimental data gathered using an in-house-built hardware prototype. A qualitative comparison between the 
simulated and real measured spectrogram shows that the SimHumalator can effectively generate high-quality microDoppler spectrograms from the motion capture data. This paper contributes to developing a radar simulator, which can help the research community augment the limited measurement training data to improve the overall classification performance and address the cold-start problem typically encountered in radar research. Furthermore, it can help simulate an electronic system without risk and efficiently perform "What if" analysis. Interested researchers can download the simulator from https://uwsl.co.uk/. While we rigorously tested the app internally and with our Beta users, there can still be a few undiscovered bugs or issues. Therefore, we believe that user feedback is crucial for developing the app.

Future simulator development will be focused on making the simulated data as close to real-world user scenarios as possible. To achieve this, we hope to incorporate both user feedback and environmental factors such as noise, multipath effects, and target and surveillance area geometrical factors. We believe this simulator can become a flexible and comprehensive tool to benchmark future algorithms and generate large volumes of realistic simulation data for the user community through a user-friendly graphical user interface (GUI). In addition, it will reduce the expense and labour involved in experimental data acquisition by other researchers and help radar and sensing communities benchmark future machine learning algorithms.

\section{ACKNOWLEDGMENTS}

This work is part of the OPERA project funded by the UK Engineering and Physical Sciences Research Council (EPSRC), Grant No: EP/R018677/1.

\section{REFERENCES}

[1] World aging population. [Online]. Available: https: //www.un.org/en/development/desa/population/publications/pdf/ ageing/WPA2017_Highlights.pdf

[2] S. Chernbumroong, S. Cang, A. Atkins, and H. Yu, "Elderly activities recognition and classification for applications in assisted living," Expert Systems with Applications, vol. 40, no. 5, pp. 1662-1674, 2013.

[3] G. Diraco, A. Leone, and P. Siciliano, "An active vision system for fall detection and posture recognition in elderly healthcare," in 2010 Design, Automation \& Test in Europe Conference \& Exhibition (DATE 2010). IEEE, 2010, pp. 1536-1541.

[4] S. Vishwakarma and S. S. Ram, "Detection of multiple movers based on single channel source separation of their micro-dopplers," IEEE Transactions on Aerospace and Electronic Systems, vol. 54, no. 1, pp. 159-169, 2017.

[5] B. Erol and M. G. Amin, "Radar data cube analysis for fall detection," in 2018 IEEE International Conference on Acoustics, Speech and Signal Processing (ICASSP). IEEE, 2018, pp. 2446-2450.

[6] V. C. Chen, F. Li, S.-S. Ho, and H. Wechsler, "Micro-doppler effect in radar: phenomenon, model, and simulation study," IEEE Transactions on Aerospace and electronic systems, vol. 42, no. 1, pp. 2-21, 2006.

[7] J. Le Kernec, F. Fioranelli, C. Ding, H. Zhao, L. Sun, H. Hong, J. Lorandel, and O. Romain, "Radar signal processing for sensing in assisted living: The challenges associated with real-time implementation of emerging algorithms," IEEE Signal Processing Magazine, vol. 36, no. 4, pp. 29-41, 2019.

[8] X. Yang, S. A. Shah, A. Ren, N. Zhao, Z. Zhang, D. Fan, J. Zhao, W. Wang, and M. Ur-Rehman, "Freezing of gait detection considering leaky wave cable," IEEE Transactions on Antennas and Propagation, vol. 67, no. 1, pp. 554-561, 2018.
[9] K. Chetty, G. E. Smith, and K. Woodbridge, "Through-the-wall sensing of personnel using passive bistatic wifi radar at standoff distances," IEEE Transactions on Geoscience and Remote Sensing, vol. 50, no. 4, pp. 1218-1226, 2011.

[10] V. C. Chen, The micro-Doppler effect in radar. Artech House, 2019.

[11] B. Tan, Q. Chen, K. Chetty, K. Woodbridge, W. Li, and R. Piechocki, "Exploiting wifi channel state information for residential healthcare informatics," IEEE Communications Magazine, vol. 56, no. 5, pp. 130 137, 2018.

[12] W. Li, B. Tan, and R. Piechocki, "Passive radar for opportunistic monitoring in e-health applications," IEEE journal of translational engineering in health and medicine, vol. 6, pp. 1-10, 2018.

[13] S. A. Shah and F. Fioranelli, "Rf sensing technologies for assisted daily living in healthcare: A comprehensive review," IEEE Aerospace and Electronic Systems Magazine, vol. 34, no. 11, pp. 26-44, 2019.

[14] Y. Lin and J. Le Kernec, "Performance analysis of classification algorithms for activity recognition using micro-doppler feature," in 2017 13th International Conference on Computational Intelligence and Security (CIS). IEEE, 2017, pp. 480-483.

[15] Y. Lin, J. Le Kernec, S. Yang, F. Fioranelli, O. Romain, and Z. Zhao, "Human activity classification with radar: Optimization and noise robustness with iterative convolutional neural networks followed with random forests," IEEE Sensors Journal, vol. 18, no. 23, pp. 9669-9681, 2018.

[16] B. Zhou, Y. Lin, J. Le Kernec, S. Yang, F. Fioranelli, O. Romain, and Z. Zhao, "Simulation framework for activity recognition and benchmarking in different radar geometries," IET Radar, Sonar and Navigation, 2020.

[17] G. E. Smith, K. Woodbridge, and C. J. Baker, "Multistatic microdoppler signature of personnel," in Radar Conference, 2008. RADAR'08. IEEE. IEEE, 2008, pp. 1-6.

[18] R. Boulic, N. M. Thalmann, and D. Thalmann, "A global human walking model with real-time kinematic personification," The visual computer, vol. 6, no. 6, pp. 344-358, 1990.

[19] G. Manfredi, P. Russo, A. De Leo, and G. Cerri, "Efficient simulation tool to characterize the radar cross section of a pedestrian in near field," Progress In Electromagnetics Research C, vol. 100, pp. 145-159, 2020.

[20] S. S. Ram and H. Ling, "Simulation of human microdopplers using computer animation data," in 2008 IEEE Radar Conference. IEEE, 2008, pp. 1-6.

[21] B. Erol and S. Z. Gurbuz, "A kinect-based human micro-doppler simulator," IEEE Aerospace and Electronic Systems Magazine, vol. 30, no. 5 , pp. $6-17,2015$

[22] A. D. Singh, S. S. Ram, and S. Vishwakarma, "Simulation of the radar cross-section of dynamic human motions using virtual reality data and ray tracing," in 2018 IEEE Radar Conference (RadarConf18). IEEE, 2018, pp. 1555-1560.

[23] "Cmu graphics lab motion capture database," online accessed on 24 December 2019. [Online]. Available: http://mocap.cs.cmu.edu/

[24] "Accad motion capture lab database," online accessed on 24 December 2019. [Online]. Available: https://accad.osu.edu/research/ motion-lab/mocap-system-and-data/

[25] M. Müller, T. Röder, M. Clausen, B. Eberhardt, B. Krüger, and A. Weber, "Documentation mocap database hdm05," Universität Bonn, Tech. Rep. CG-2007-2, June 2007.

[26] E. F. Knott, J. F. Schaeffer, and M. T. Tulley, Radar cross section. SciTech Publishing, 2004.

[27] "Phase-space motion capture system," online accessed on 10 August 2020. [Online]. Available: https://www.phasespace.com/

[28] X. Microsoft, "Kinect sensor," 2021. [Online]. Available: www.xbox. com/en-US/kinect

[29] F. Restuccia, "Ieee 802.11 bf: Toward ubiquitous wi-fi sensing," arXiv preprint arXiv:2103.14918, 2021.

[30] D. Dhulashia, M. Ritchie, S. Vishwakarma, and K. Chetty, "Human micro-doppler signature classification in the presence of a selection of jamming signals." IEEE Radar Conference (RadarCon), 2021.

[31] Y. Kim and H. Ling, "Human activity classification based on microdoppler signatures using a support vector machine," IEEE Transactions on Geoscience and Remote Sensing, vol. 47, no. 5, pp. 1328-1337, 2009.

[32] R. Ricci and A. Balleri, "Recognition of humans based on radar microdoppler shape spectrum features," IET Radar, Sonar \& Navigation, vol. 9, no. 9, pp. 1216-1223, 2015.

[33] G. Li, R. Zhang, M. Ritchie, and H. Griffiths, "Sparsity-driven microdoppler feature extraction for dynamic hand gesture recognition," 
IEEE Transactions on Aerospace and Electronic Systems, vol. 54, no. 2, pp. 655-665, 2017.

[34] S. Vishwakarma and S. S. Ram, "Dictionary learning with low computational complexity for classification of human micro-dopplers across multiple carrier frequencies," IEEE Access, vol. 6, pp. 29793-29805, 2018.

[35] S. S. Keerthi, S. K. Shevade, C. Bhattacharyya, and K. R. Murthy, "A fast iterative nearest point algorithm for support vector machine classifier design," IEEE transactions on neural networks, vol. 11, no. 1, pp. 124$136,2000$.

[36] C. Tang, S. Vishwakarma, W. Li, R. Adve, S. Julier, and K. Chetty, "Augmenting experimental data with simulations to improve activity classification in healthcare monitoring," in accepted for IEEE Radar Conference (RadarConf21). IEEE, 2021.

[37] Ni usrp 2921. [Online]. Available: http://sine.ni.com/nips/cds/view/ $\mathrm{p} / \mathrm{lang} / \mathrm{en} / \mathrm{nid} / 212995$

[38] S. Vishwakarma, C. Tang, W. Li, K. Woodbridge, R. Adve, and K. Chetty, "Gan based noise generation to aid activity recognition when augmenting measured wifi radar data with simulations." IEEE International Conference on Communications, 2021. 